\title{
O IMPACTO DO PADRÃO HISTOLÓGICO DE INVASÃO NAS CARACTERÍSTICAS CLÍNICO-PATOLÓGICAS E NA EVOLUÇÃO DO CARCINOMA EPIDERMÓIDE INICIAL DA LARINGE
}

\author{
INVASION HISTOLOGICAL PATTERN AND CLINICAL-PATHOLOGICAL \\ PRESENTATION AND OUTCOME OF EARLY SQUAMOUS CELL CARCINOMA OF \\ THE LARYNX
}

\author{
Otávio Alberto Curioni ${ }^{1}$ \\ Marcos Brasilino de Carvalho ${ }^{2}$ \\ Marilene Paladino Rosa ${ }^{3}$ \\ José Carlos Baraúna Neto ${ }^{4}$ \\ Abrão Rapoport, TCBC-SP ${ }^{5}$
}

\begin{abstract}
RESUMO: Objetivo: Avaliar o padrão de invasão na interface tumor-hospedeiro com alguns parâmetros que caracterizam a lesão primária com a intenção de caracterizar essa informação como fator influente na adequação terapêutica de pacientes com lesões iniciais na laringe. Métodos: Análise retrospectiva das fichas médicas e revisão histológica dos blocos dos espécimes cirúrgicos de 49 pacientes portadores de carcinoma epidermóide inicial da laringe (T1 e T2), tratados no Serviço de Cirurgia de Cabeça e Pescoço do Complexo Hospitalar Heliópolis - SP, entre 1977 e 1997. O padrão da invasão da neoplasia no tecido subjacente foi definido no ponto de invasão tumoral (critério de Bryne) em quatro categorias (padrão de 1 a 4), e a avaliação estatística descritiva das variáveis (macroscopia T, N, necrose tumoral e padrão de invasão) foi feita para a associação das mesmas, através dos testes de hipóteses do Qui-quadrado e para as freqüências com Teste Exato de Fisher, com 95\% de significância. Resultados: A relação do padrão de invasão e da microscopia demonstrou, para as lesões infitrativas, dez casos $(41,6 \%)$, no padrão 1 , oito $(33,3 \%)$ no padrão 2 e seis $(25,0 \%)$ no padrão 3 . Para as lesões vegetantes, seis $(24,0 \%)$ padrão $1,15(60,0 \%)$ padrão 2 e nove $(16,0 \%)$ padrão 3. Quanto à região anatômica, para a glote tivemos dez $(29,4 \%)$ padrão $1,17(50,0 \%)$ padrão 2 e sete $(20,6 \%)$ padrão 3. Para a supraglote, seis $(40,0 \%)$ padrão 1 , seis $(40,0 \%)$ padrão 2 e dez $(23,3 \%)$ padrão 3. Para N+, quatro $(66,7 \%)$ padrão 1 e dois $(33,3 \%)$ padrão 2. Para a necrose tumoral, quando ausente, tivemos $13(31,7 \%)$ padrão 1, $19(46,3 \%)$ padrão 2 e nove $(21,9 \%)$ padrão 3. Quando presente, três $(37,5 \%)$ padrão 1 , quatro $(50,0 \%)$ padrão 2 e um $(12,5 \%)$ padrão 3. Finalmente, o prognóstico não tem qualquer relação com o padrão de invasão, sendo nos pacientes vivos, $14(87,5 \%)$ padrão 1,16 $(72,5 \%)$ padrão 2 e sete $(70,0 \%)$ padrão 3. Conclusão: A diversidade dos resultados e a não confirmação estatística impõem a necessidade de se desenvolver estudos prospectivos, com maior série de casos, para conclusões mais confiáveis em relação ao padrão de invasão em lesões classificadas como iniciais, inclusive utilizando-se tumores primários de outras localizações.
\end{abstract}

Descritores: Laringe; Carcinoma epidermóide; Padrão de invasão; Histopatologia.

\section{INTRODUÇÃO}

A escolha do tratamento para o carcinoma epidermóide da laringe é freqüentemente influenciada pela sua localização inicial e extensão e pela probabilidade em apresentar metástase para linfonodos regionais. Em geral, tu- mores com elevada incidência de metastatização são tratados com procedimentos mais radicais do que aqueles que apresentam baixa incidência, mesmo nas lesões ditas iniciais. Por essa razão, estudos que tratam sobre a probabilidade de metastatização neoplásica são importantes nas considerações terapêuticas.

1. Mestre em Cirurgia de Cabeça e Pescoço - CPG Hospital Heliópolis

2. Doutor em Cirurgia pela FCM da UNICAMP

3. Serviço de Anatomia Patológica do Hospital Heliópolis

4. Residente em Cirurgia de Cabeça e Pescoço

5. Coordenador CPG Hospital Heliópolis - Livre-docente pela FMUSP

Recebido em 11/04/2001

Aceito para publicação em 19/07/2001

Trabalho realizado no Serviço de Cirurgia de Cabeça e Pescoço do Complexo Hospitalar Heliópolis, São Paulo, Brasil. 
Entre outros parâmetros, o padrão de invasão na interface tumor-hospedeiro tem sido valorizado como um indicador prognóstico no câncer de cabeça e pescoço, particularmente para os tumores extensos, ou seja, extensão local considerada avançada. As lesões cujo padrão de invasão se faz em cordões celulares (infiltrativos), por exemplo, teriam uma natureza mais agressiva que aquelas com padrão bem delimitado (pushing) ${ }^{1}$.

Aparentemente, quanto mais a célula tumoral perder suas características de adesão intercelular maiores serão as diferenças com o tecido normal e maior será a possibilidade de que êmbolos tumorais se desprendam e viabilizem o desenvolvimento de metástases regionais ou à distância. Obviamente, quando estamos diante de tumores avançados, com invasão extensa de estruturas vizinhas e metástases clinicamente evidentes, esta discussão é desprovida de utilidade. Entretanto, quando estamos tratando de pacientes com tumores iniciais de laringe, onde a possibilidade de cura é elevada, é essencial que a decisão sobre o primeiro tratamento seja a mais fundamentada possível. Tradicionalmente, este fundamento é feito sobre dados da observação clínica e de exame anatomopatológico ${ }^{2}$.

Com base nesses fatos, este estudo correlaciona o padrão de invasão na interface tumor-hospedeiro com alguns parâmetros que caracterizam a lesão primária como sítio, tamanho e metástase linfonodal regional em 49 tumores iniciais da laringe. Essa informação poderia ser utilizada na adequação do tratamento e no seguimento racionalizado pós-operatório de pacientes tratados com cirurgia por lesões primárias classificadas como iniciais.

A realização deste estudo foi autorizada pela Comissão de Ética em Pesquisa do Complexo Hospitalar Heliópolis (Protocolo ${ }^{\circ}$ 76).

\section{MÉTODOS}

A partir de análise retrospectiva dos prontuários e revisão histológica das lâminas preparadas a partir do recorte dos blocos dos espécimes cirúrgicos, este trabalho procurou correlacionar o padrão de invasão na interface tumor-hospedeiro e outras características clínico-patológicas de 49 pacientes portadores de carcinoma epidermóide inicial da laringe tratados no Serviço de Cirurgia de $\mathrm{Ca}$ beça e Pescoço do Complexo Hospitalar Heliópolis, SP, no período de setembro de 1977 e dezembro de 1997. Como pré-requisito para ingresso no estudo os pacientes deveriam ser portadores de carcinoma epidermóide, confirmado através de exame anatomopatológico de respectivas biópsias; serem virgens de tratamento prévio; a neoplasia primária estadiada em fase inicial (T1 ou T2 classificação TNM 1997) e terem sido submetidos a tratamento cirúrgico.

A idade dos pacientes variou entre 29 e 75 anos, com média e mediana de 58 e 59 anos, respectivamente. Para pacientes com lesões glóticas, a idade média foi 60,8 anos e a mediana 60 anos; e para as lesões supraglóticas esses valores foram 55,6 e 65,5 anos, respectivamente. Em ambos os grupos predominaram pacientes entre as sexta e sétima décadas de vida.
Características relacionadas à localização, à extensão e ao aspecto macroscópico da lesão primária eram descritas nos prontuários médicos e registradas graficamente sob a forma de esquemas. Dos achados de laringoscopia direta e indireta foram revistas a localização, extensão, macroscopia e a presença ou não de linfonodos suspeitos de serem metastáticos.

$\mathrm{Na}$ eventualidade de haver discordâncias entre os dados morfológicos assinalados nos períodos pré e transoperatórios relativos ao sítio e à extensão da lesão primária foram considerados como verdadeiros aqueles referidos na descrição do achado cirúrgico.

Dos blocos parafinados do material obtido da peça cirúrgica, cortes histológicos foram corados pela hematoxilina-eosina (HE) e foram revistas para detalhamento das características referentes ao padrão de invasão na interface tumor-hospedeiro, presença de desmoplasia, infiltração perineural, embolização angiolinfática, presença de necrose tumoral e grau de diferenciação celular.

O padrão de invasão da neoplasia no tecido subjacente foi definido no ponto de maior invasão tumoral segundo critério de Bryne et al. ${ }^{2}$ (1992), que o classificam em quatro categorias:

- padrão 1: tumor tem bordas bem delimitadas, pushing (Figura 1);

- padrão 2: as margens de progressão do tumor infiltram como cordões sólidos ou faixas (Figura 2);

- padrão 3: tumor cujas margens infiltrativas contêm grupos pequenos ou cordões de células (Figura 3);

- padrão 4: a interface tumor-hospedeiro mostra marcada dissociação celular em pequenos grupos ou células isoladas.

Análises estatísticas descritivas foram utilizadas para as variáveis macroscópicas ${ }^{1}, \mathrm{~T}, \mathrm{~N}$, necrose tumoral e padrão de invasão. A associação entre as variáveis foi obtida através dos testes de hipótese Qui-quadrado ou Exato de Fisher para as frequiências, dependendo da casuística, com 95\% de significância.

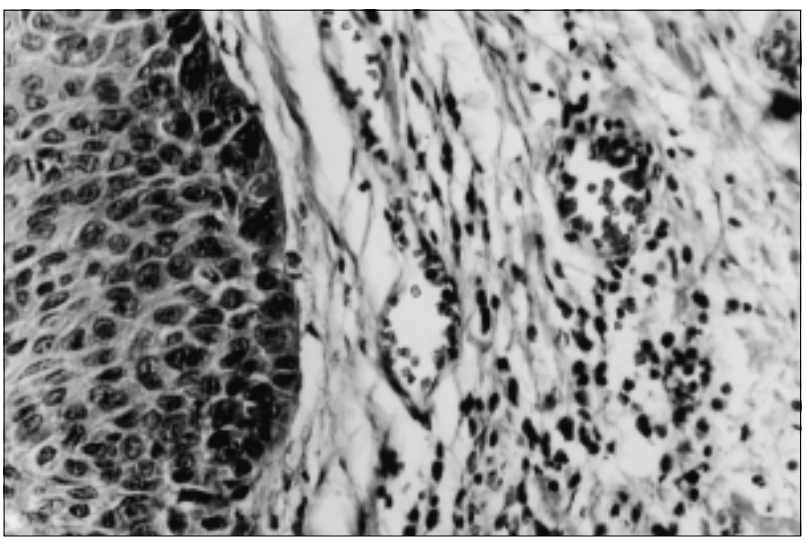

Figura 1 - Fotomicrografia de padrão 1 de invasão. Coloração H.E., aumento de $400 \mathrm{X}$. 


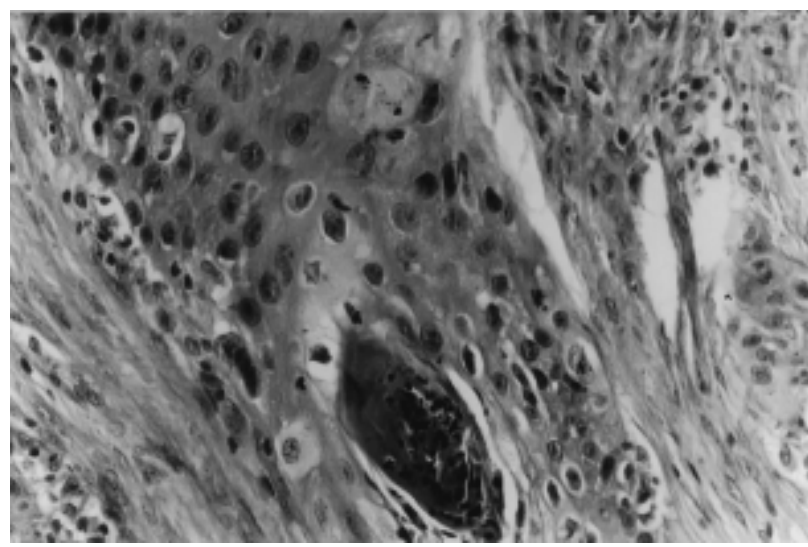

Figura 2 - Fotomicrografia de padrão 2 de invasão. Coloração H.E., aumento de $400 \mathrm{X}$.

\section{RESULTADOS}

O padrão de invasão, quando correlacionado com a característica macroscópica da lesão primária, revelou que, embora sem significância estatística, para as lesões infiltrativas predominou o padrão 1 e para as vegetantes o padrão 2 (Tabela 1).

Da mesma forma, em relação ao sítio anatômico da lesão primária a distribuição do padrão de invasão não apresentou diferença significativa para os tumores da glote e da supraglote (Tabela 2).

A categoria T da classificação TNM também não apresentou significância comparada ao padrão de invasão (Tabela 3), assim como para a categoria N. Porém, dos seis casos de metástases cervical positiva, quatro apresentaram padrão 1 e nenhum com metástase foi observada no padrão 3 (Tabela 4).

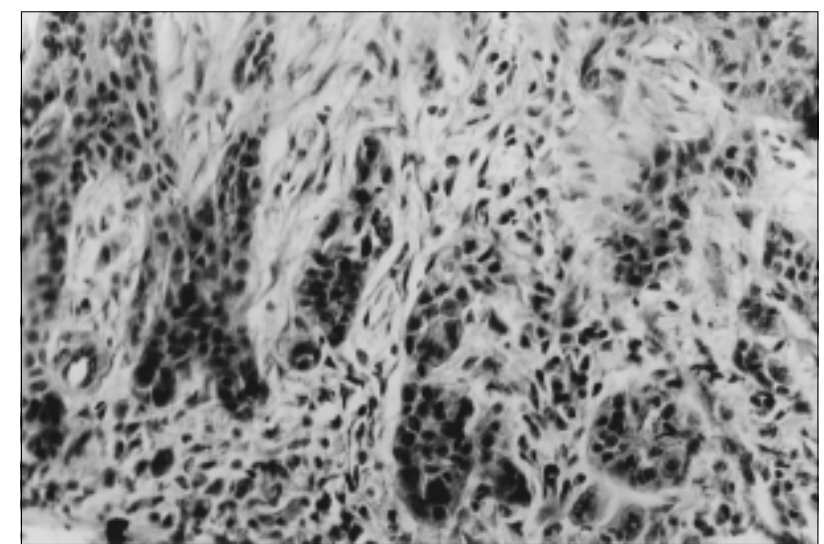

Figura 3 - Fotomicrografia de padrão 3 de invasão. Coloração H.E., aumento de $200 \mathrm{X}$.

O padrão de invasão correlacionado à presença de necrose na lesão primária não apresentou associação significativa (Tabela 5).

Nenhum dos 49 casos apresentou o padrão 4 de infiltração.

Na análise da evolução dos pacientes não obtivemos interferência significativa quando comparamos cada padrão individualmente, porém o índice de óbitos relacionados ao câncer da laringe foi duas vezes maior no grupo de pacientes com padrões mais dissociados (Tabela 6).

\section{DISCUSSÃO}

A maioria dos tumores malignos é, obviamente, invasiva e esta invasividade não reconhece limites anatômicos normais. Seguindo o desenvolvimento das metástases, é o fator mais constante que caracteriza um tumor maligno. A invasividade do câncer permite que ele pene-

Tabela 1

Distribuição dos pacientes segundo padrão de invasão e macroscopia da lesão primária

\begin{tabular}{l|c|c|c|c}
\hline Macroscopia & Padrão 1 & Padrão 2 & Padrão 3 \\
$N(\%)$ & $N(\%)$ & $\begin{array}{c}\text { Total } \\
N(\%)\end{array}$ \\
\hline Infiltrativa & $10(41,6 \%)$ & $8(33,3 \%)$ & $6(25 \%)$ & $24(100 \%)$ \\
Vegetante & $6(24,8 \%)$ & $15(60,0 \%)$ & $4(16,0 \%)$ & $25(100 \%)$ \\
\hline
\end{tabular}

$P=0,190$

Tabela 2

Distribuição dos pacientes segundo padrão de invasão e região anatômica da lesão primária

\begin{tabular}{l|c|c|c|c}
\hline Sítio & Padrão 1 & Padrão 2 \\
$N(\%)$ & $N(\%)$ & $\begin{array}{c}\text { Padrão } 3 \\
N(\%)\end{array}$ & $\begin{array}{c}\text { Total } \\
N(\%)\end{array}$ \\
\hline Glote & $10(29,4 \%)$ & $17(50,0 \%)$ & $7(20,6 \%)$ & $34(100 \%)$ \\
Supraglote & $6(40,0 \%)$ & $6(40,0 \%)$ & $4(16,0 \%)$ & $15(100 \%)$
\end{tabular}

$p=0,850$ 
Tabela 3

Distribuição dos pacientes por variável T e padrão de invasão

\begin{tabular}{|c|c|c|c|c|}
\hline Variável & $\begin{array}{c}\text { Padrão } 1 \\
N(\%)\end{array}$ & $\begin{array}{c}\text { Padrão } 2 \\
N(\%)\end{array}$ & $\begin{array}{c}\text { Padrão } 3 \\
N(\%)\end{array}$ & $\begin{array}{c}\text { Total } \\
N(\%)\end{array}$ \\
\hline 1 & $8 \quad(32,0 \%)$ & $11 \quad(4,0 \%)$ & $6(24 \%)$ & 25 \\
\hline 2 & $8 \quad(33,3 \%)$ & $12(50,0 \%)$ & $4 \quad(16,7 \%)$ & 24 \\
\hline Total & 16 & 23 & 10 & 49 \\
\hline
\end{tabular}

$p=0,869$

Tabela 4

Distribuição dos pacientes segundo padrão de invasão e estádio N

\begin{tabular}{l|c|c|r|r}
\hline Variável & Padrão 1 & Padrão 2 & Padrão 3 & Total \\
$N(\%)$ & $N(\%)$ & $1 \%)$ \\
\hline$(-) \mathrm{N} 0$ & $12(27,9 \%)$ & $21(48,8 \%)$ & $10(23,3 \%)$ & $43(100,0 \%)$ \\
$(+) \mathrm{N}+$ & $4(66,7 \%)$ & $2(33,3 \%)$ & 0 & $6(100,0 \%)$ \\
\hline Total & 16 & 23 & 10 & 49 \\
\hline
\end{tabular}

$p=0,195$

Tabela 5

Distribuição dos pacientes segundo padrão de invasão e necrose tumoral

\begin{tabular}{|c|c|c|c|c|}
\hline Necrose & $\begin{array}{c}\text { Padrão } 1 \\
N(\%)\end{array}$ & $\begin{array}{c}\text { Padrão } 2 \\
N(\%)\end{array}$ & $\begin{array}{c}\text { Padrão } 3 \\
N(\%)\end{array}$ & $\begin{array}{c}\text { Total } \\
N(\%)\end{array}$ \\
\hline (-) Ausente & $13(31,7 \%)$ & $19(46,4 \%)$ & $9 \quad(21,9 \%)$ & $41 \quad(100 \%)$ \\
\hline (+) Presente & $3 \quad(37,5 \%)$ & $4 \quad(50,0 \%)$ & $1(12,5 \%)$ & $8 \quad(100 \%)$ \\
\hline Total & 16 & 23 & 10 & 49 \\
\hline
\end{tabular}

$p=1,0$

Tabela 6

Evolução dos pacientes estudados

\begin{tabular}{l|c|c|c|c}
\hline Padrão & $\begin{array}{c}\text { MOAS } \\
N(\%)\end{array}$ & $\begin{array}{c}\text { MOCA } \\
N(\%)\end{array}$ & $\begin{array}{c}\text { OOO } \\
n(\%)\end{array}$ & $\begin{array}{c}\text { Total } \\
\text { (\%) }\end{array}$ \\
\hline 1 & - & $2(12,5 \%)$ & $14(87,5 \%)$ & 16 \\
2 & $1(20,0 \%)$ & $6(25,5 \%)$ & $16(72,5 \%)$ & 23 \\
3 & $1(10,0 \%)$ & $2(20,0 \%)$ & $7(70,0 \%)$ & 10 \\
\hline Total & 2 & 10 & 37 & 49 \\
\hline
\end{tabular}

MOAS = morto assintomático

MOCA = morto por câncer

tre nos vasos sangüíneos e/ou linfáticos, usualmente no nível capilar, e esta infiltração progressiva é acompanhada de invasão e destruição do tecido vizinho. Em geral, a fronteira é pouco demarcada em relação ao tecido normal vizinho e falta um plano de clivagem bem definido.

Por causas ignoradas, alguns tumores podem desenvolver uma aparente cápsula fibrosa envoltória resultante de uma frente de compressão sobre as estruturas normais adjacentes. Portanto, parece claro que tumores constituídos por blocos volumosos com bordas coesas e extensas seriam menos propensos a invadir canais vasculares, especialmente pequenos espaços capilares. Em contrapartida, tumores que se caracterizam por maior perda da adesividade intercelular e que invadam os tecidos vizinhos, 
como pequenos agregados celulares ou como células isoladas, seriam mais aptos para invadir o tecido normal do hospedeiro a fim de ganhar acesso ao compartimento vascular e, portanto, disseminar.

A razão para esse efeito pode estar relacionada à distribuição da membrana basal (colágeno tipo IV e laminina) nestes padrões de invasão ${ }^{3}$. Assim, a infiltração neoplásica em cordões irregulares ou células isoladas poderia representar uma invasão ativa dentro do estroma tecidual. Em contraste, cordões largos e/ou coesos de células neoplásicas conservariam a expressão da membrana basal separando o tumor invasivo do estroma hospedeiro.

Nesta série não encontramos variação significativa quando comparamos o padrão de invasão com a característica macroscópica da lesão primária, apesar da literatura ser contraditória. Enquanto alguns autores encontraram lesões com padrão coeso de infiltração predominantemente na supraglote e, além disso, as lesões cujo padrão de infiltração era composto de pequenos agregados celulares eram pobremente diferenciadas ${ }^{4}$, outros relacionaram o padrão coeso de infiltração com pior prognóstico ${ }^{5}$.

Em relação ao sítio de origem da lesão primária, foram encontrados predominantemente lesões com padrão coeso de infiltração na supraglote ${ }^{4,6,7}$. Nossos resultados evidenciaram um predomínio de lesões com padrão coeso ou pouco dissociado de infiltração independentemente se a lesão era de glote ou supraglote, e estas lesões são usualmente exofíticas, mais diferenciadas e menos invasivas do que as lesões com padrão de infiltração dissociado ${ }^{6,8}$. Embora sem confirmação estatística, nossos achados são destoantes daqueles relatados na literatura. As lesões com padrão celular coeso, em $62,5 \%$ dos casos, apresentavam características mais infiltrativas do que vegetantes, apresentaram distribuição por grau histológico semelhantes.
Esta distribuição pode ser devida ao pequeno número de casos por nós estudados, ou seja, uma amostra maior poderá trazer resultados diferentes.

O estádio T relacionado ao padrão de invasão também não mostrou variação significativa, onde, tanto as lesões T1 como as lesões T2 apresentaram, com maior freqüência, o padrão celular de infiltração pouco dissociado, apesar de autores, em lesões T1 de soalho oral, encontrarem maioria de lesões com padrão celular coeso de infiltração ${ }^{9}$. Porém, outros autores demonstraram que em lesões T1 e T2 de língua oral predominaram lesões com padrão de infiltração com poucas células dissociadas e padrão com limites pouco nítidos na interface tumor-hospedeiro ${ }^{5}$.

Embora não tenhamos encontrado confirmação estatística, na nossa série houve predomínio de metástase cervical associada a lesões cujo padrão de invasão era coeso; a literatura constatou maiores taxas de metástases cervicais associadas a lesões primárias cujo padrão de invasão predominava o mais dissociado, em detrimento do padrão coeso ${ }^{10}$.

É possível que esses resultados conflitantes sejam reflexos da limitada casuística estudada ou, para os tumores iniciais, o comportamento do padrão de invasão na interface tumor hospedeiro seja diverso daquele encontrado em tumores avançados.

A diversidade dos resultados encontrada e a falta de comprovação estatística impõem a necessidade de se desenvolver estudos prospectivos, com maior série de casos, para conclusões mais confiáveis em relação ao padrão de invasão na interface tumor hospedeiro em tumores iniciais. Estas questões talvez possam ser esclarecidas estudando-se a influência destas variáveis para tumores primários de outros sítios anatômicos.

\begin{abstract}
Background: Our objective is to evaluate the invasion pattern (ip) of the initial laryngeal tumours with some parameters that characterize the primary lesion, as an important factor of therapeutic response. Methods: This is a retrospective analysis of medical records and histology blocks of surgical specimens of 49 patients with carcinoma epidermoid of the larynx (T1 and T2), treated in the Head and Neck Surgery Service from Heliópolis Hospital - SP, between 1977 and 1997. The tumour interface invasion pattern was defined as tumoral invasion point (Bryne criterion) through 4 categories ( 1 to 4 pattern), and the descriptive statistical evaluation of variables (macroscopy, T, $N$, tumoral necrosis and invasion pattern) was perfomed through their association, using the $K$ Square Text, and for frequencies, the Exact Fisher Text (95\% of Interval Confidence). Results: The invasion pattern (ip) and the macroscopic relation showed for infiltratives lesions, ip 1 in 10 cases (41,6\%),ip 2 in 8 cases $(24,0 \%)$ and ip, 3 in 6 cases (25,0\%). For exophytic lesions, 6(24,0\%) for ip 1, 15(60,0\%) for ip 2 and 9(16,0\%) for ip 3. For anatomic region, for glottis 10(29,4\%) for ip 1, 17(50,0\%) for ip 1,6(40,0\%) for ip 2 and 3(20,0\%) for ip 3. For N0, 12(27,9\%) i.p. 1, 21(48,8\%) i.p. 2. For the absence tumoral necrosis, 13(31,7\%) ip 1,19(46,3\%) ip 2 and 9(21,9\%) ip 3; when diagnosed, 3(37,5\%) for ip 1,4(50\%) for ip 2 and 1(12,5\%) for ip 3. Finally, in this material, no relation was stablished between ip and survival (for patients alive, 14(87,5\%) for ip 1, 16(72,5\%) for ip 2 and 7(70,0\%) for ip 3. Conclusion: Our results showed a diversity of results and imposes the need for prospective studies, with larger number of cases to establish invasion pattern in lesions classified as initials, besides using primary tumours of other locations.
\end{abstract}

Key Words: Larynx; Squamous cell carcinoma; Invasion pattern; Histopathology. 


\section{REFERÊNCIAS}

1. Crissman JD, Gluckman JL, Cummings AG. Prognostic value of histopathologic parameters in squamous cell carcinoma of the oropharynx. Cancer, 15:299501,1984 .

2. Bryne M, Koppang HS, Lilleng R et al. Malignancy grading of the deep invasive margins of oral squamous cell carcinoma has high prognostic value. J Pathol, 1992, 166:375-381.

3. Walder F. Distribuição da membrana basal e sua correlação com variáveis antomoclínicas: um estudo himuno-histoquímico da disposição do colágeno tipo iv em carcinomas epidermóides de via aerodigestiva superior. Dissertação [Mestrado - Curso de Pós-Graduação em Cirurgia de Cabeça e Pescoço do Complexo Hospitalar Heliópolis] São Paulo, 1997.

4. McGavran MH, Bauer WC, Ogura JH. The incidence of cervical lymph node metastases from epidermoid carcinoma of the larynx and their relationship to certain characteristcs of the primary tumor. Cancer 14:5566, 1961.

5. Ravasz LA, Hordijik GJ, Slootweg PJ et al. Uni- and multivariate analysis of eight indications for post-operative radiotherapy and their significance for local-regional cure in advanced head and neck cancer. J Laryngol Otol 107:437-40,1993.

6. McDonald TJ, De Santo LW, Weiland LH. Supraglottic larynx and its pathology as studied by whole laryngeal sections. Laryngoscope 86:635-48,1976.

7. Barona de Guszman R, Martorell MA, Basterra J et al. Prognostic value of histophathological parameters in 51 supraglottic squamous cell carcinoma. Laryngoscope 103:358-40,1993.
8. Kirschner JA, Som ML. Clinical and histological observations on supraglotic cancer. Ann Otol Rhinol Laryngol 80:638-45, 1971.

9. Gluckman JL, Pavelic ZP, Welkoborsky HJ et al. Prognostic indicators for squamous cell carcinoma of the oral cavity: A clinicopathologic correlation. Laryngoscope, 1977, 107:1239-1244.

10. Yamamoto E, Myiakawa A, Kohana G. Mode of invasion and lymph node metastasis in squamous cell carcinoma of the oral cavity. Head Neck Surg 6:93847,1984 .

Endereço para correspondência:

Prof. Dr. Abrão Rapoport

Praça Amadeu Amaral, 47 - cj. 82 - Paraíso

01327-010 - São Paulo-SP

Tel.: (011) 289-6229 / 287-4347

E-mail: cpgcp.hosphel@attglobal.net 